\title{
Outcome of Cloverleaf Locking Plate Fixation for Femoral Neck Fractures in Young Adults
}

\author{
Ismail HD, Ph.D, Phedy*, MD, Oktavian Irawadi Purba*, MD, Bambang Gunawan, MD, \\ Djoko Simbardjo, MD
}

Department of Orthopaedic and Traumatology, Medical Faculty University of Indonesia - Cipto Mangunkusumo Hospital, Jakarta, Indonesia

*Department of Orthopaedic and Traumatology, Medical Faculty University of Indonesia - Cipto Mangunkusumo

Hospital, Jakarta, Indonesia

\begin{abstract}
Femoral neck fractures in young adults remain a major problem in orthopaedics. Although treatments using cannulated screws and sliding hip screws are widely accepted, they pose significant clinical challenges. In the present study, we evaluated the outcome of treatment using cloverleaf locking plate fixation (augmented with the addition of addition of nonvascularised autologous bone grafts for neglected fracture). Fourteen patients were investigated in this cross-sectional study. Union times and Harris hip scores were analysed by independent T-test based on displacement (displaced vs. nondisplaced) and type (acute vs. neglected) of fracture. No difference in union time was found between acute and neglected groups and between nondisplaced and displaced fractures. Harris hip scores were similar between acute and neglected groups and between non- and displaced group. Cloverleaf locking plate fixation is a viable alternative method for stable fixation of femoral neck fractures in young adults.
\end{abstract}

Key Words:

Femoral neck fracture, young adult, cloverleaf locking plate, Harris hip score, union rate

\section{INTRODUCTION}

Fractures of the femoral neck in young adults have been regarded as a challenge in orthopaedics ${ }^{1} 2$. Due to the scant blood supply, these fractures carry high risks of non-union and avascular necrosis. Non-union rates for femoral neck fractures reach $33 \%$ while the avascular necrosis accounts for $16 \%$ of complications ${ }^{1}$. In developing countries, these complications are even higher, and such injuries are often neglected for several months due to poverty, ignorance, lack of facilities, and faith in traditional bonesetters. Adding to the complexity of their management, fractures of femoral neck have a 20 to $36 \%$ reoperation rate within two years of the first internal fixation and 6 to $18 \%$ after hemiarthroplasty ${ }^{1}$.
Cannulated screws, sliding hip screws, and proximal femoral locking plates are three widely-used internal fixation devices for femoral neck fracture in young adults ${ }^{3}$. Although cannulated and sliding hip screws allow dynamic compression of the fracture site during weight bearing, they cannot prevent secondary limb shortening due to the lateralization of the head or neck fragment from gliding along the screw ${ }^{4}$. Both also carry a high risk of mechanical fracture, especially cutout of the implant from the femoral head ${ }^{5,6}$. Other limitations of the sliding hip screw include the large amount of bone removed, the possibility of damaging the femoral head blood supply, and the inability to control rotation ${ }^{7}$. Proximal femoral locking plates have been evaluated in a limited study ${ }^{8}$. Although showing promising results, such findings may not be applicable in the young population as the study was conducted in an elderly cohort with a mean subject age of 75-years-old and had neither control nor randomization.

In our centre, we encounter both cases of acute and neglected fractures of femoral neck. We have used cloverleaf locking plates to manage femoral neck fractures since 2002. The cloverleaf locking plate is similar in design to the proximal femoral locking plate. However, only three screws are needed for fixation of the femoral neck, with the cloverleaf plate, mimicking requirement of cannulated screws (triangular configuration). Other advantages of the plate include its wide availability and affordable price. The outcomes of clinical management of the cloverleaf locking plate are evaluated in the present study.

\section{MATERIALS AND METHODS}

In this cross-sectional study, we reviewed all patients age 15to 55-years-old who were treated between January 2003 and July 2010 with a diagnosis of femoral neck fracture and treated using the cloverleaf locking plate. Informed consents were obtained from all patients before their data were included in this study. The use of the cloverleaf locking plate 
to manage femoral neck fracture in our centre was approved by the institutional ethics committee. The stainless steel implants utilised in this study were patented (Indonesia patent number ID 0006435) and manufactured by one of the authors (University Indonesia, Jakarta, Indonesia).

Nonunion rate, union time and functional outcome based on Harris hip scores ${ }^{9}$ at the time of union were recorded and analysed based on the displacement (displaced vs. nondisplaced) and type (acute vs. neglected) of the fracture by independent t-test. All fractures were fixed using the cloverleaf locking plate (Figure 1). Nonvascularised autologous bone grafts were added to augment fracture in neglected cases.

\section{RESULTS}

Fourteen patients, seven male and seven female, were included in this study (Table I). The age of the patients ranged from 17- to 55-years-old. Eight fractures were nondisplaced (Garden Grade I and II) and six were displaced (Garden Grade III and IV). One patient had nonunion despite treatment with sliding hip screws. Six patients had neglected fracture. The remainder of neglected patients went to bone setters before presenting to our centre. The neglect delay ranged from three to six months with the average of $5 \pm 1.3$ months. (Table I)

Despite two fractures, which underwent surgery four months previous to presenting at our centre, all fractures in this study united. Union was achieved in 7.5 \pm 2.4 months (Figure 2). When analysed according to type, union was achieved in $7.7 \pm 2.5$ and $7.2 \pm 2.5$ months for the acute and neglected groups respectively. Union time for the nondisplaced group
Table I: Patient Characteristics

\begin{tabular}{|lc|}
\hline Characteristics & No. \\
\hline Age*(years) & $29.9 \pm 11.8$ \\
Sex & 6 \\
Female & 8 \\
Male & \\
Mechanism of trauma & 12 \\
Road vehicle accidents & 1 \\
Fall from height & 1 \\
Sport injury & \\
Garden Grade & 1 \\
Grade I & 5 \\
Grade II & 1 \\
Grade III & 7 \\
Grade IV & \\
Type of fracture & 8 \\
Acute & 6 \\
Neglected & \\
\hline
\end{tabular}

* mean \pm standard deviation

was $7.4 \pm 2.4$ and $7.4 \pm 2.7$ months for the displaced group. These differences were not statistically significant $(\mathrm{p}=0.77$; $95 \% \mathrm{CI}-2.7-3.7$ for acute vs. neglected group and 0.93; $95 \% \mathrm{CI}-3.2-3.3$ for nondisplaced vs. displaced group).

The average functional outcome (Harris hip score) was $85.8 \pm 5.1$. The scores of acute and neglected group were $85.5 \pm 5.3$ and $86.2 \pm 5.5$ respectively. Harris hip scores of the nondisplaced group were $84.3 \pm 2.2$ and $87.8 \pm 4.1$ for the displaced group. These differences were not statistically significant $(p=0.74 ; 95 \% \mathrm{CI}-7.6-6.3$ for acute vs. neglected group and $\mathrm{p}=0.60 ; 95 \% \mathrm{CI}-9.7-2.6$ for nondisplaced vs. displaced group).

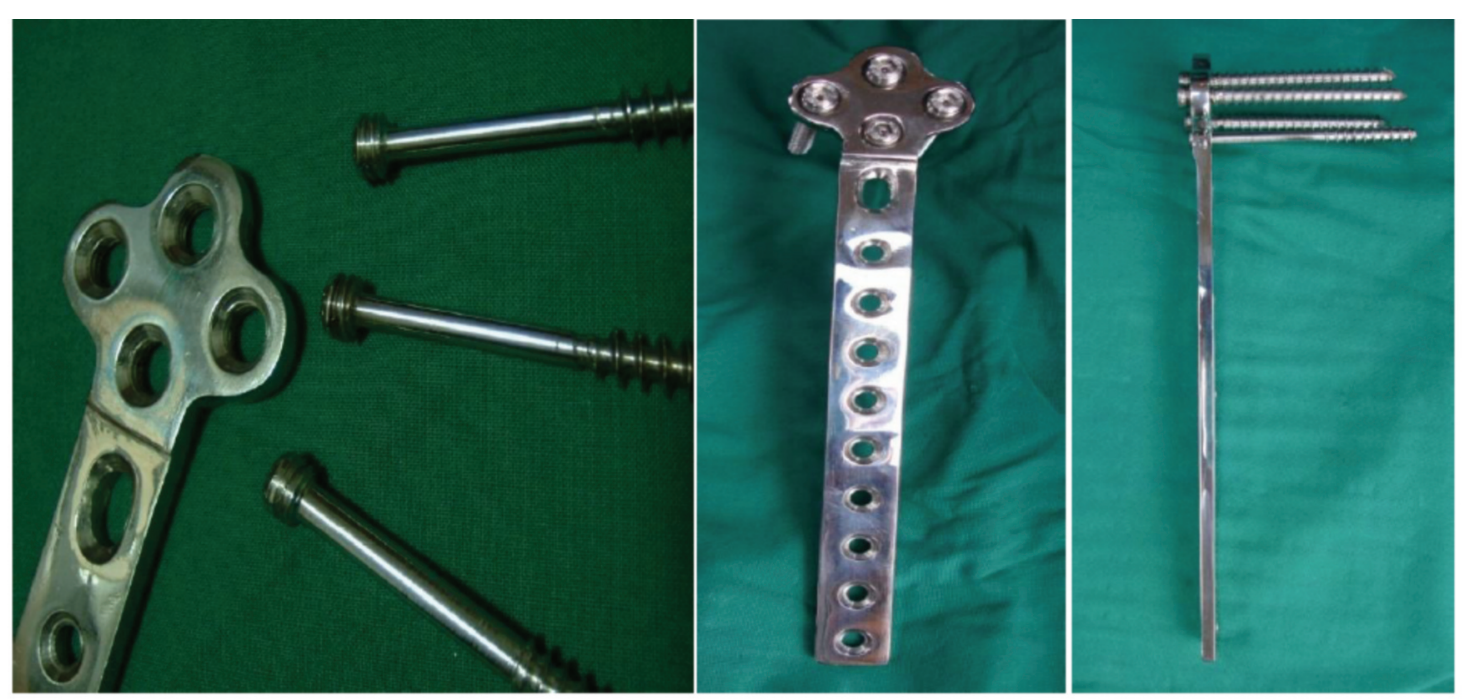

Fig. 1: Cloverleaf locking plate. Left: construct of the plate before application of the locking screws. Middle: anteroposterior view of the plate with screws inserted. Right: lateral view of the plate with screws inserted. 


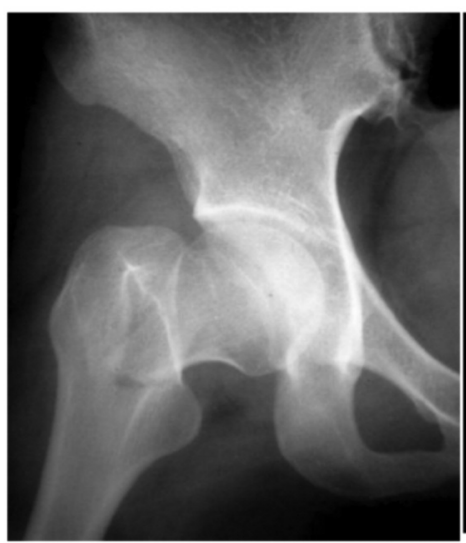

(a)

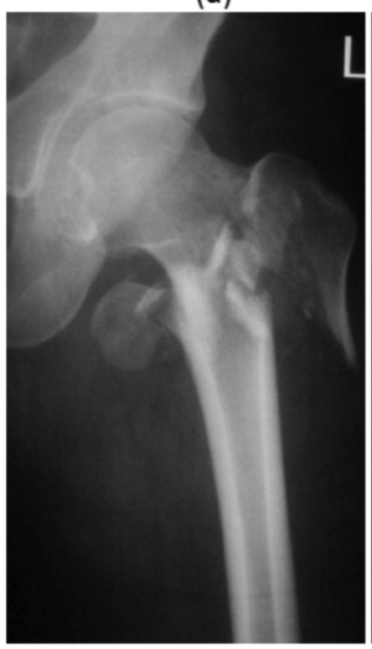

(a)

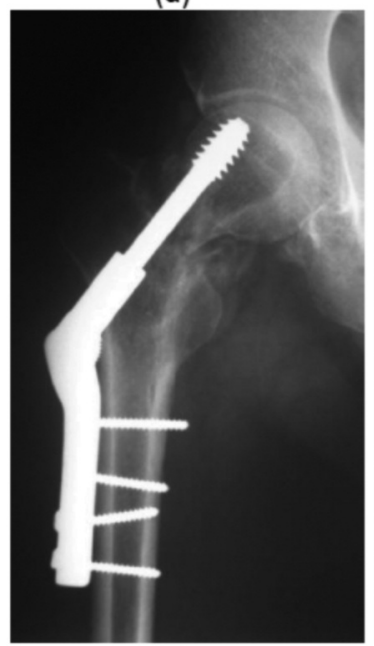

(a)

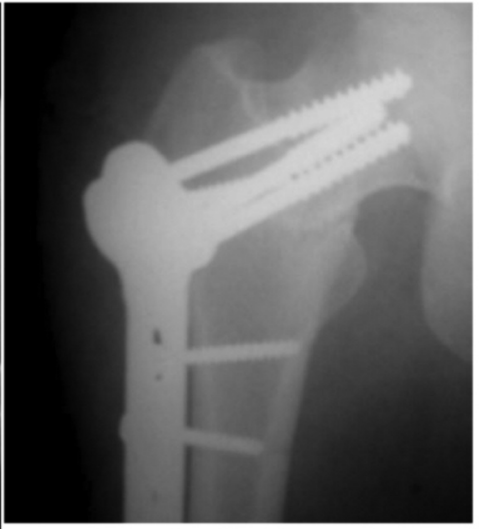

(b)

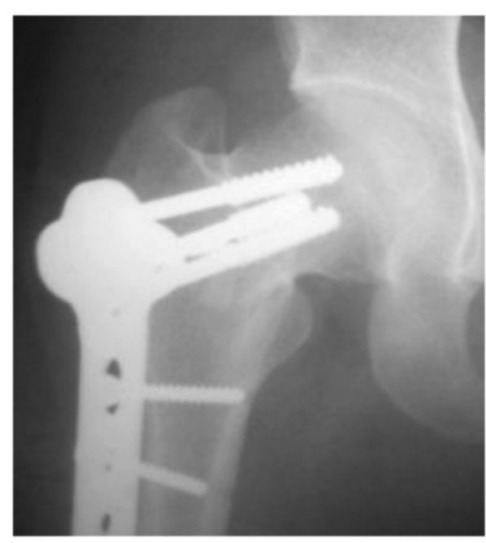

(c)

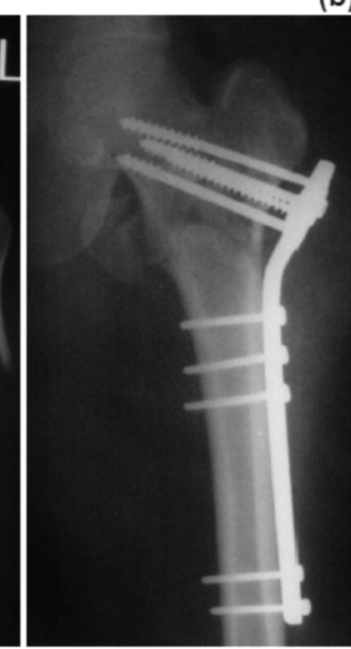

(b)

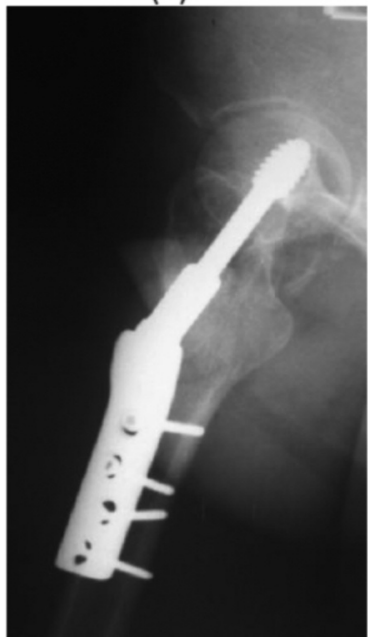

(b)
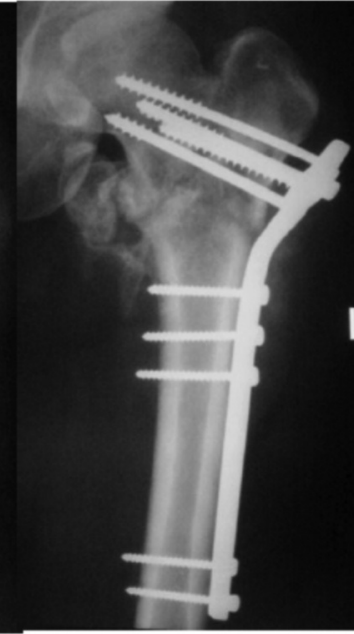

(c)

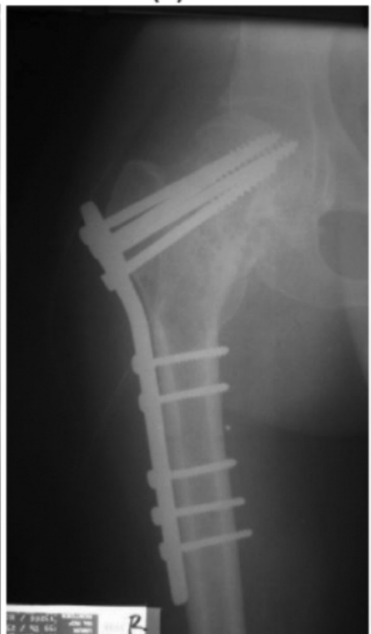

(c)

L

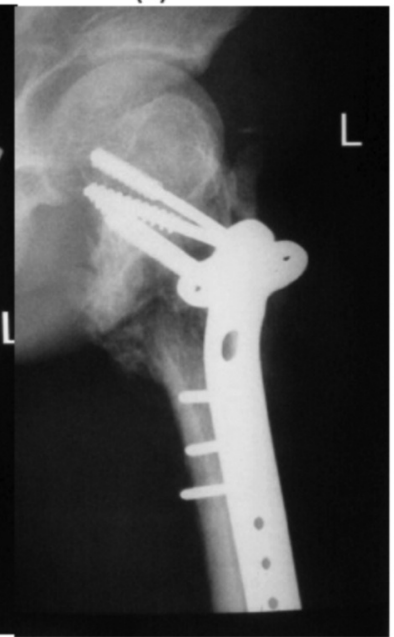

(d)

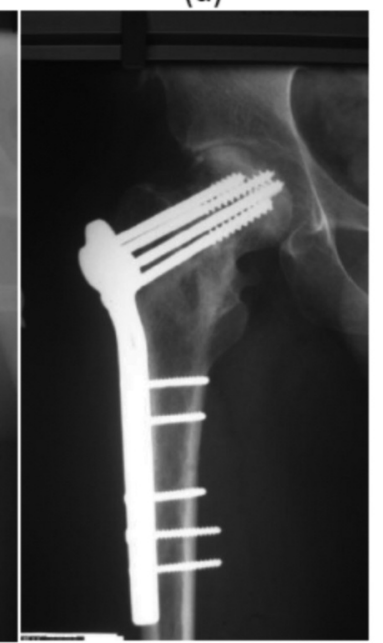

(d)

Fig. 2: Treatment of femoral neck fracture with cloverleaf locking plate fixation. Top: Acute Garden grade II fracture (a) treated without bone graft (b). Union was achieved after 6 months and (c) the Harris hip score was 88. Middle: Garden grade IV fracture untreated for six months (a) augmented with bovine demineralized bone matrix (b). Union was achieved after 6 months (c, d) and Harris functional score was 94. Bottom: Nonunion fracture of Garden grade II fracture treated initially with sliding hip screws. After 6 months of follow up, no union was seen $(a, b)$. The fixation was replaced and the fracture was augmented with nonvascularized autologous bone graft(c). Union was achieved after 12 months (d) with Harris hip score of 84. 


\section{DISCUSSION}

This study reviewed the results of 14 cases of femoral neck fractures in young adults that were treated with cloverleaf locking plate. High-energy trauma was the predominant cause of femoral neck fractures in this study. Some patients presented with neglected fractures. All cases of neglected fracture presented with non-union after several months.

Bone quality has been reported as the single most predictor of stability of bone implant units in femoral neck fractures ${ }^{10}$. However, it should be noted that such results were obtained from femora of female cadavers aged above 60-years-old. Therefore, the findings may not be applicable to young populations.

Gotfried $^{11}$ emphasized maintenance of reduction of the proximal segment within femoral head in addition to midsegment and distal fixation at the lateral trochanteric wall as the key to unimpaired fracture healing. The proximal segment is responsible for load-bearing and controlling caudal shift and varus tilt of the femoral head. The midsegment bridges the fracture site while the distal segment provides stable anchorage in the proximal femur.

The cloverleaf locking plate was designed and developed by one of the authors (DS). The leaf of the plate allows screws insertion within the femoral head, while the bar of the plate holds the middle and distal fragment. The cloverleaf plate acts as a fixed-angle device, allowing some degree of compression of the fracture site and prevents additional femoral neck shortening. The locking screws provide point contact fixation making the device suitable for use in treatment of pathological and osteoporotic fracture. Locking of the screw to the side plate provides better torsional resistance. Resistance is further enhanced further by the multiple axis fixation design. The design increases the projected area of fixation in the femoral head and consequently the strength of fixation.

One of the major problems associated with femoral neck fractures is nonunion. One study reported that the nonunion rate for displaced femoral neck fractures was as high as $33 \% \%^{1,12}$. In a retrospective study, Butt et al. reported nonunion rates of displaced femoral neck fracture to be $8 \%$, and that $33 \%$ of those nonunion cases fail to unite even after secondary procedures ${ }^{13}$. The age of patients in the Butt and colleagues study ranged from 20- to 55-years-old. In our study, all but two fractures united. Since nonunion is defined as the persistence of a visible fracture line at a minimum of 6 months after the index procedure ${ }^{13}$, the two cases were excluded from analyses of union rate given that their surgery took place within the previous four months. Nonunion rate are reported to be higher in displaced compared to nondisplaced fractures ${ }^{12}$. However, we couldn't confirm this finding since there were no cases of nonunion in our study.

Roshan et al. reported lower union rates than our finding with the usage of two cannulated screws augmented with a vascularized graft ${ }^{14}$. However, the average union time in the Roshan study for neglected fracture was 19.2 weeks, which is superior to ours. Differences in type of graft presented difficulties for analyses about whether the superiority was due to the type of fixation. Moreover, they only included patients with displaced femoral neck fracture.

Varying methods had been used to assess functional outcomes in femoral neck fractures. Some authors evaluated pain, walking status and need for gait aids as the outcome, while others used the Judet point system to grade the disability $^{2,13}$. Similar to Roshan et al, we used Harris hip scores to assess the functional outcome and found comparable results ${ }^{14}$.

The present findings provide evidence to advocate fpr the use of cloverleaf locking plate fixation in the management of femoral neck fracture in young adults. Of note however, the current study was limited by the small numbers of patients in the study sample. Moreover, we did not assess functional score preoperatively and therefore do not have data regarding postoperative improvement. Another welldesigned prospective study with larger sample and longer follow up should be conducted to confirm these preliminary results.

\section{CONCLUSION}

Cloverleaf locking plate fixation serves as a viable alternative to provide stable fixation for femoral neck fractures in young adults. It results in a high union rate and 


\section{REFERENCES}

1. Lu-Yao GL, Keller RB, Littenberg B, Wennber JE. Outcomes after displaced fractures of the femoral neck. A meta-analysis of one hundred and six published reports. J Bone Joint Surg Am. 1994; 76(1): 15-25.

2. Davidovitch RI, Jordan CJ, Egol KA, Vrahas MS. Challenges in the treatment of femoral neck fractures in the nonelderly adult. J Trauma. 2010; 68(1): 236-42.

3. Miyamoto RG, Kaplan KM, Levine BR, Egol KA, Zuckerman JD. Surgical management of hip fractures: An evidenced-based review of the literature. I: Femoral neck fractures. J Am AcadOrthop Surg. 2008; 16: 596-607.

4. Hasenboehler EA, Agudelo JF, Morgan SJ, Smith WR, Hak DJ, Stahel PFl. Treatment of complex proximal femoral fractures with the proximal femur locking compression plate. Orthopaedics. 2007; 30(8): 618-23.

5. Pervez H, Parker MJ, Vowler S. Prediction of fixation failure after sliding hip screw fixation. Injury. 2004; 35: 994-8.

6. Bout CA, Cannegieter DM, Juttmann JW. Percutaneous cannulated screw fixation of femoral neck fractures: the three point principle. Injury. 1997; 28(2): 135-9.

7. Ly TV, Swiontkowski MF. Management of femoral neck fractures in young adults. Indian J Orthop. 2008; 42(1): 3-12.

8. Parker MJ, Stedtfeld HW. Internal fixation of intracapsular hip fractures with a dynamic locking plate: initial experience and results for 83 patients treated with a new implant. Injury. 2010; 41: 348-51.

9. Harris WH. Traumatic arthritis of the hip after dislocation and acetabular fractures: treatment by mold arthroplasty: An end-result study using a new method of result evaluation. J Bone Joint J Surg Am. 1969; 51: 737-55.

10. Clark DI, Crofts CE, Saleh M. Femoral neck fracture fixation: comparison of a sliding screw with lag screws. J Bone Joint Surg Br. 1990; 72: 797-800.

11. Gotfried Y, Cohen B, Rotem A. Biomechanical evaluation of the percutaneous compression plating system for hip fractures. $J$ Orthop Trauma. 2002; 16(9): 644-50.

12. Haidukewych G, Rothwell WS, Jacofsky DJ, Yotchia ME, BerryDJ. Operative treatment of femoral neck fractures in patients between the ages of fifteen and fifty years. J Bone Joint Surg Am. 2004; 86(8):1711-6.

13. Butt MF, Dhar SA, Gani N, Farooq M, Mir MR, Halwal MA, et al. Delayed fixation of displaced femoral neck fractures in younger adults. Injury Int J Care. Injured. 2008; 39:238-43.

14. Roshan A, Ram S. Early return to function in young adults with neglected femoral neck fractures. Clin Orthop Relat Res. 2006; 447: $152-7$. 